\title{
Méthodes de mesure de débits dans les écoulements diphasiques. Diflux et Optimix
}

\author{
Yves Lecoffre \\ YLec Consultants $\left({ }^{1}\right)$
}

\section{Les besoins}

La mesure des débits dans les écoulements diphasiques pose un grand nombre de problèmes pratiques, liés en particulier aux aspects suivants :

- les types d'écoulement rencontrés sont extrêmement variables en fonction des dimensions des tuyauteries, de leur inclinaison, des rapports de débits de chacune des phases, des glissements relatifs et des très grandes variations des propriétés physiques des écoulements tout au long des réseaux ;

- on est souvent amené à effectuer ces mesures au moyen des capteurs utilisés traditionnellement en écoulements monophasiques, turbines, organes déprimogènes, débitmètres dits à effet Coriolis :

- il est, en général nécessaire d'ajouter à ces mesures à caractère global, une ou plusieurs mesures supplémentaires permettant de caractériser l'état thermodynamique du système, température, pression ou concentration d'une phase dans l'autre.

Il n'existe pas de système de débitmétrie universel utilisable en écoulements diphasiques. Seul, peut-être, le débitmètre RMN développé à l'Ecole Supérieure de Physique et Chimie pourra prétendre à cette fonction lorsqu'il sera industrialisé. Pratiquement, l'appareil le mieux adapté à telle ou telle situation dépend de plusieurs facteurs, selon les objectifs précis de la mesure.

Dans le cas d'écoulements turbulents, YLec Consultants a développé deux concepts répondant à des besoins spécifiques, la débitmétrie gaz-liquide et plus précisément la débitmétrie eau-vapeur avec l'appareil Diflux et la débitmétrie liquide-liquide dans le cas de fluides non miscibles dont la base est le mélangeur Optimix.

Pour la débitmétrie gaz-liquide, on sépare les deux phases avant de réaliser leur mesure de manière indépen-

(') Allée des Dauphins, 38330 Saint Ismier, France. Tél.: (33) 76521863 . dante. Dans le cas de la débitmétrie liquide-liquide, on mélange au contraire intimement les deux phases avant de réaliser la mesure du débit total et celle des concentrations relatives des deux corps.

\section{Débitmétrie gaz-liquide}

\subsection{Les besoins}

Le développement du concept de débitmètre séparateur compact a été lancé à la demande d'un grand distributeur de vapeur saturée pour chauffage urbain. L'objectif était de réaliser une gestion centralisée d'un réseau, ce qui impliquait la mise en place de débitmètres fiables. Les appareils utilisés jusqu'alors étaient des appareils déprimogènes, diaphragmes et venturi, fonctionnant en général en régime d'écoulement bloqué, complétés par une mesure de température qui donnait accès à l'état thermodynamique du fluide.

Le besoin exprimé était de disposer d'un débitmètre permettant de mesurer indépendamment l'eau et la vapeur et leur température afin de calculer le débit d'enthalpie passant au compteur.

Les contraintes particulières de ce type de mesure sont essentiellement la très grande variabilité des consommations selon des cycles journaliers, hebdomadaires et saisonniers. S'y ajoutent dans une moindre mesure des variations de pression.

Par ailleurs, l'objectif était de mettre en œuvre un débitmètre suffisamment précis pour pouvoir effectuer un véritable comptage de calories en privilégiant la mesure de la phase vapeur. Il était d'autre part souhaitable d'éviter de trop grandes pertes de pression au passage de l'appareil.

\subsection{Les principes}

L'appareil dont nous avons développé le concept est constitué des éléments suivants :

\section{Flow measurement methods in two-phase flows. Diflux and Optimix}

Flow measurement in two-phase flows is difficult. YLec Consultants has developped two instruments : Diflux for " water-vapour » and the Optimix mixer for fluid-fluid flow measurement in the case of non miscible fluids. 


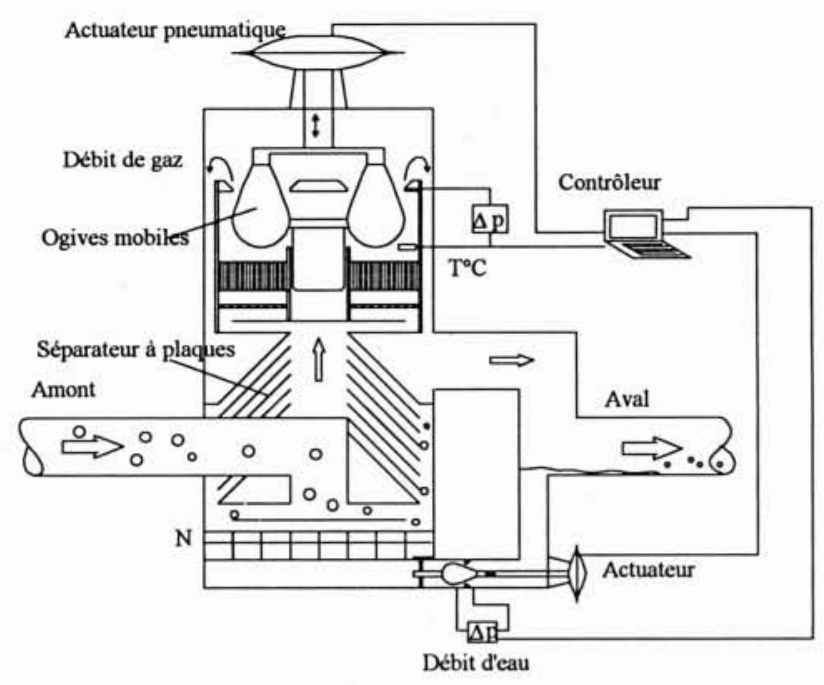

1. Diflux : principes.

- un séparateur de phases compact gravitaire ou cyclonique qui permet d'obtenir des phases les plus pures possibles quel que soit le taux de vide dans la tuyauterie amont ; - une mesure de température et de pression permettant de connaître l'état thermodynamique de la vapeur. Dans le cas de vapeur toujours saturée, il suffit de connaître l'une ou l'autre de ces grandeurs;

- un débitmètre vapeur déprimogène à tuyères multiples mobiles. Ce débitmètre a une section au col variable et fonctionne normalement à perte de charge constante. Le débit dépend de la position de la tuyère :

— un débitmètre liquide. On mesure ce débit, soit par un appareil déprimogène à section variable, soit au moyen d'un appareil plus traditionnel, un débitmètre électromagnétique par exemple ;

- une vanne de régulation permettant de maintenir le niveau de liquide dans le séparateur à une valeur spécifiée. Dans le cas d'un débitmètre déprimogène à section variable, cette fonction de contrôle est assurée par le débitmètre lui-même.

Après réalisation de ces mesures, les deux phases sont à nouveau mélangées dans la tuyauterie aval.

\subsection{Les séparateurs}

Il est intéressant de réaliser des séparateurs compacts pour ce type d'application. Nous avons donc envisagé deux solutions, un séparateur gravitaire et un séparateur cyclone. Le choix de l'un ou l'autre de ces appareils dépend du cahier des charges précis de la mesure et, en particulier, de la gamme de variation des paramètres et des débits.

Le séparateur que nous avons envisagé en version de base est représenté sur la figure l. Il s'agit d'un système gravitaire axisymétrique comportant des éléments permettant la séparation et la coalescence des gouttelettes d'eau. En général, les gouttes de liquide sont assez grosses, de diamètre supérieur à $100 \mu \mathrm{m}$.

La disposition particulière des éléments séparateurs inclinés à environ $45^{\circ}$ vers l'axe de l'appareil permet de réduire notablement l'encombrement de cet élément. L'objectif sera, en général, d'éviter les réentraînements de gouttes de diamètre important, par exemple supérieur à $100 \mu \mathrm{m}$. On mettra donc en œuvre des séparateurs sièges d'un écoulement laminaire en vapeur dans la gamme de températures considérées.

En ce qui concerne les dimensions de l'appareil, on peut envisager un temps de séjour compris entre 5 et $10 \mathrm{~s}$ au débit volumique maximal pour réaliser la fonction de séparation selon la granulométrie de coupure choisie.

L'écoulement dans l'appareil de séparation doit être très régulier pour que la surface libre de liquide soit la plus calme possible. Elle sert, en effet, à la commande de la vanne de régulation du débit de liquide.

L'emploi d'un séparateur gravitaire compact est intéressant lorsque les variations de débit sont importantes. II suffit en effet de le dimensionner pour les débits les plus élevés pour couvrir toute la gamme possible d'utilisation. Par ailleurs, la perte de charge d'un séparateur gravitaire peut être très faible, typiquement égale à deux fois la pression dynamique de l'écoulement dans la tuyauterie amont.

La figure 2 donne une variante de l'appareil dans laquelle le séparateur gaz-liquide est simplement incliné.

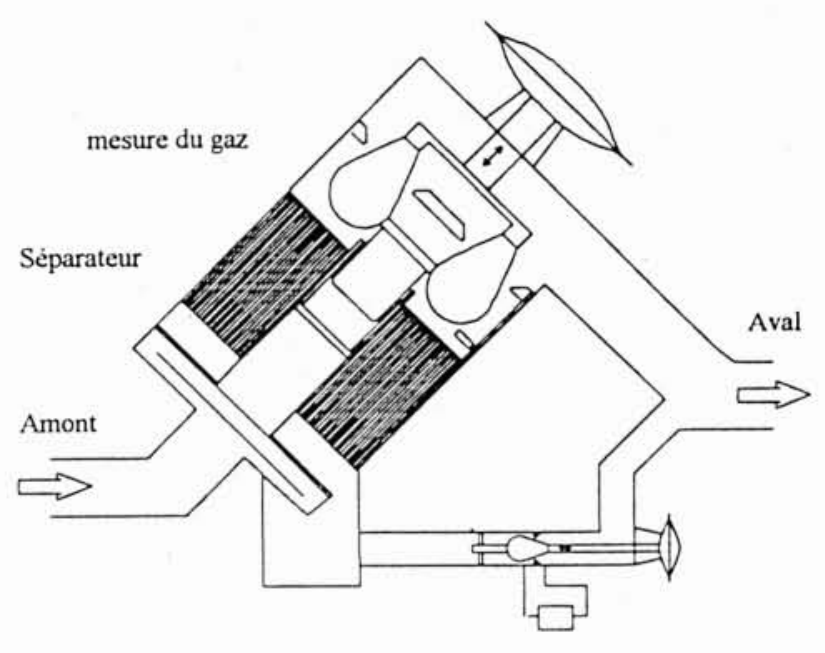

mesure du liquide

\section{Diflux, version inclinée.}

On peut également imaginer de mettre en place un cyclone afin de diminuer l'encombrement de l'appareil. Dans ce cas, le dimensionnement sera réalisé au débit maximal afin d'obtenir un diamètre de coupure des gouttelettes admissible en fonction de la perte de charge disponible. Lorsque le débit diminue, on risque de voir diminuer l'efficacité de séparation et de voir le cyclone se transformer en séparateur gravitaire pour les débits les plus faibles.

\subsection{Les tuyères de mesure de gaz}

Les figures 1 et 2 donnent des schémas de la section de mesure du débit de gaz. Cette section de mesure peut être constituée d'une seule tuyère pour les appareils de petite taille ou de plusieurs, cas que nous avons représenté pour 


\section{MESURE DE DÉBITS EN ÉCOULEMENTS DIPHASIQUES}

les tuyauteries de grand diamètre, typiquement supérieur à $150 \mathrm{~mm}$.

Le bloc de tuyères est alimenté par la vapeur qui provient du séparateur. L'écoulement de vapeur est mis en forme par une grille suivie d'un bloc de nids d'abeille. On obtient ainsi un débit homogène exempt de rotations résiduelles.

La vapeur pénètre alors dans la section de mesure. On aura, au préalable mesuré sa température et éventuellement sa pression afin de connaître son état thermodynamique.

On mesure alors la différence de pression entre l'amont et le col de l'une des tuyères. La vapeur est ensuite évacuée vers la tuyauterie aval. La conception proposée, avec retour axisymétrique permet de réaliser un ensemble compact.

Dans un premier mode d'utilisation, la connaissance de cette différence de pression et de la position de la tuyère permettent de déterminer le débit de vapeur passant dans l'appareil.

On préfère travailler à différence de pression constante et mesurer la position de la tuyère. Ceci permet d'obtenir une précision relative constante sur les valeurs des débits. Afin de limiter les pertes de charge de l'appareil, on fonctionnera le plus souvent en écoulement subsonique. Pratiquement, on peut envisager une perte de charge de 5000 à 10000 Pa pour les applications les plus courantes.

La régulation de la position de la vanne est assurée par un automate spécifique qui effectue l'ensemble des mesures et calculs nécessaires.

On obtient donc des courbes de fonctionnement telle celle de la figure 3 . On a représenté la différence de pression dans la vanne en fonction du débit pour une vanne à une seule tuyère de diamètre égal à $50 \mathrm{~mm}$. Entre les points $\mathrm{A}$ et $\mathrm{B}$ de la courbe, pour le fonctionnement à $150{ }^{\circ} \mathrm{C}$ pris comme exemple, la différence de pression est constante et le débit varie entre $14 \mathrm{l} / \mathrm{s}$ et $180 \mathrm{l} / \mathrm{s}$, soit une dynamique de 12,8 , avec une précision relative de mesure constante. En deçà, la précision de la mesure devient moins bonne. Au delà, la perte de charge augmente suivant les lois quadratiques classiques.

$\triangle \mathrm{pkPa}$

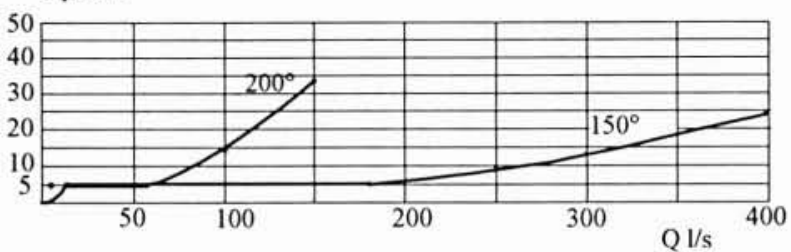

3. Diagramme de fonctionnement simplifié d'un Diflux, côté vapeur.

On constate que la gamme de fonctionnement global, s'étend, pour une température de vapeur donnée, sur une dynamique d'environ 40 .

\subsection{Mesure du débit de liquide}

La perte de charge de la section de mesure de la vapeur doit être suffisamment élevée pour que le liquide puisse être évacué à l'aval de l'appareil. Il est, en effet nécessaire de contrôler son niveau à la sortie du séparateur au moyen d'une vanne de régulation. Ceci nécessite une différence de pression de l'ordre de celles que nous avons proposées ci-dessus.

En général, dans les applications à la débitmétrie de vapeur saturée, il n'est pas nécessaire de connaître le débit avec une très grande précision. On peut donc se contenter d'utiliser la perte de charge de la vanne de régulation et sa position pour en déduire le débit. Dans d'autres applications nécessitant une meilleure précision sur la mesure de débit de liquide, on mettra en œuvre un organe de mesure du même type que celui que nous utilisons pour la vapeur. Cet organe de mesure pourra en général être utilisé comme vanne de réglage.

\section{Mesure de débits et mélange}

\subsection{Problèmes et méthode}

La débitmétrie de mélanges liquide-liquide peut être effectuée assez facilement par utilisation d'une méthode déprimogène, pour peu que les deux composants soient suffisamment mélangés. L'écoulement est alors considéré comme homogène. On suppose en particulier que les glissements entre phases sont négligeables.

Si les liquides sont intimement mélangés, on peut, de plus, considérer que les taux de présence de chacune des phases sont les mêmes en tous points de l'écoulement.

On peut alors déterminer le débit de chacune des phases en effectuant une mesure du débit global, $Q$. caractérisé par la masse volumique du mélange, $\rho_{m}$ et une analyse des proportions de chacune des phases à partir d'un prélèvement.

On écrit alors les équations suivantes :

$$
\begin{aligned}
Q & =K \sqrt{\frac{\Delta p}{\rho_{m}}} \\
\rho_{m} & =\alpha \rho_{1}+(1-\alpha) \rho_{2}
\end{aligned}
$$

$\alpha$ étant le taux de présence de la phase 1 dans le mélange. En écoulement homogène, on peut écrire :

$$
\alpha=\frac{Q_{1}}{Q}
$$

$\rho_{1}$ et $\rho_{2}$ sont respectivement les masses volumiques des corps 1 et $2, Q_{1}$ le débit volumique de la phase $\mathrm{n}^{\circ} 1$.

On déduit donc de ces deux mesures, débit total et masse volumique du mélange, ou taux de présence, le débit de chacune des phases.

L'une des difficultés majeures de cette méthode de mesure est liée à la qualité du mélange. Dans le cas le plus courant des tuyauteries horizontales, celui-ci doit en effet être parfaitement homogène sur toute la hauteur de la conduite. Ceci implique pratiquement la mise en œuvre d'un mélangeur efficace. Ce mélangeur doit pouvoir créer un mélange homogène dans toute la gamme des débits de l'une ou l'autre des phases, tout en n'engendrant pas de pertes de charge rédhibitoires au débit maximal de l'installation.

Pour ce type d'applications, YLec Consultants, en collaboration avec SPRETEC a développé un mélangeur doté d'un étage d'émulsionnement réglable, cas particulier du système OPTIMIX. 


\subsection{OPTIMIX}

La figure 4 représente un mélangeur OPTIMIX placé sur une conduite transportant un écoulement stratifié.



4. OPTIMIX, application au dosage.

Les deux fluides pénètrent entre les deux premières viroles, qui constituent l'étage dit de dosage ou de macromélange. Ils s'y répartissent de manière homogène selon le périmètre. Cette opération est quasiment indépendante du nombre de Reynolds, et ceci jusqu'à des valeurs d'environ 1000 .

Les fluides montent ensuite dans le jeu interviroles et pénètrent dans les orifices de micromélange. Ces orifices sont calculés pour que la perte de charge de l'appareil ne soit pas excessive au débit maximal de la canalisation et que la granulométrie des globules en suspension soit suffisamment faible pour qu'ils ne puissent pas se déposer sous l'action de la gravité avant la section de mesure.

On détermine la granulométrie des globules en appliquant les lois de HinZE et Kolmogorov, qui donnent le diamètre $D_{\max }$ des plus gros d'entre eux. $D_{\max }$ est fonction de la dissipation de puissance par unité de masse de mélange $\varepsilon$ et de la tension superficielle $\sigma$ entre les phases. Pratiquement, pour un appareil Optimix, elle est donnée par :

$$
D_{\max }=0.725 \varepsilon^{-0.4}\left(\frac{\rho}{\sigma}\right)^{-0.6}
$$

$\rho$ étant la masse volumique de la phase non dispersée.

Lorsque le débit diminue, la puissance dissipée par unité de volume varie en $V^{3}$. Le diamètre des globules a donc naturellement tendance à augmenter. Les risques de ségrégation sont donc beaucoup plus importants à faible débit.

L'appareil OPTIMIX est muni d'un étage de micromélange réglable, qui permet de compenser ce phénomène en diminuant la dimension des orifices. On diminue ainsi le volume de dissipation, tout en augmentant le coefficient de perte de charge. Il suffit de régler l'ouverture des orifices et d'appliquer l'équation de KoLMOGORov pour obtenir une granulométrie adaptée à chaque débit. On peut alors mettre en œuvre la technique de mesure explicitée en 3.1 .

Cet appareil est utilisable pour effectuer le comptage de tout écoulement multiphasique liquide-liquide. Citons une application possible pour les terminaux de pétrole. On cherche, en général, à connaître la quantité d'eau transportée avec le brut, qui peut représenter jusqu'à $10 \%$ du débit total. Pour une telle mesure, on peut mettre en œuvre un OPTIMIX dont le diamètre intérieur sera égal à environ deux diamètres de conduite. Le poste de mesure peut être, quant à lui, placé immédiatement derrière l'appareil.

Des essais ont été récemment menés au CENG, qui montrent la très bonne efficacité de l'appareil dans une configuration voisine de celle que nous avons décrite cidessus. Par ailleurs, on montre que la granulométrie en écoulement diphasique suit effectivement les lois de HiNZE et Kolmogorov. Ceci a été par exemple mesuré sur des mélangeurs OPTIMIX utilisés dans la fabrication d'émulsions de bitumes liquides utilisés dans les revêtements de routes.

\section{Conclusion}

Ces deux exemples d'appareillages de mesure de débits en écoulements diphasiques illustrent le fait qu'il n'y a pas de solution universelle et que l'on doit le plus souvent se contenter de méthodes monophasiques ou quasi monophasiques.

Le système Diflux utilise un séparateur très compact. II est surtout applicable pour les écoulements gaz-liquide, qui constituent des milieux dont les phases sont faciles à séparer. Son système de contrôle de section de passage permet de couvrir une dynamique de l'ordre de 50, suffisante dans beaucoup d'applications pratiques et, en tout cas très supérieure à celle des appareils déprimogènes traditionnels. On peut espérer une précision sur les débits de chacune des phases de l'ordre de quelques pour cent. Bien entendu, plus le séparateur sera de grande taille et meilleure sera cette précision.

Le débitmètre utilisant le mélangeur OPTIMIX est d'un principe quasi opposé. Plutôt que de séparer les phases, opération très délicate en écoulement liquide-liquide, on cherche au contraire à les mélanger suffisamment intimement pour rendre le mélange homogène. Il suffit alors de mesurer le débit du mélange et les proportions des différentes phases dans celui-ci pour obtenir une mesure fiable. Le mélangeur Optimix permet de concevoir des stations de mesure très compactes. Son système de réglage des granulométries permet d'autre part de limiter les pertes de charge au strict nécessaire pour une gamme de débits donnés. Spretec et YLec Consultants disposent en effet d'une procédure d'industrialisation des mélangeurs qui consiste à dimensionner des appareils optimisés pour chaque cas d'application. 